Clinical trial

\title{
A multicenter randomized clinical trial evaluating interleukin-2 activated hematopoietic stem cell transplantation and post-transplant IL-2 for high risk breast cancer patients
}

\author{
Claudine Isaacs ${ }^{1}$, Rebecca Slack ${ }^{2}$, Edmund Gehan ${ }^{2}$, Karen Ballen $^{3}$, Ralph Boccia ${ }^{4}$, Ellen \\ Areman $^{1}$, Ruthie Kramer ${ }^{5}$, Daniel F. Hayes ${ }^{1,6}$, Herbert Herscowitz ${ }^{7}$, and Marc Lippman ${ }^{1,6}$ \\ ${ }^{1}$ Division of Hematology and Oncology, Lombardi Comprehensive Cancer Center, Washington, DC, 20057, USA; \\ ${ }^{2}$ Division of Biostatistics and Bioinformatics, Department of Oncology, Lombardi Comprehensive Cancer Center, \\ Washington, DC, USA; ${ }^{3}$ Bone Marrow Transplant Program, University of Massachusetts Memorial Medical Center, \\ Lake Avenue, Worcester, Massachusetts, USA; ${ }^{4}$ Stem Cell Transplant Program, Holy Cross Hospital, Silver Spring, \\ Maryland, USA; ${ }^{5}$ Clinical Research Management Office, Lombardi Comprehensive Cancer Center, Washington, DC, \\ USA; ${ }^{6}$ Department of Internal Medicine, University of Michigan Health and Hospital System, Ann Arbor, MI, USA; \\ ${ }^{7}$ Microbiology and Immunology, Georgetown University Medical Center, Washington, DC, USA
}

Key words: breast cancer, immunotherapy, interleukin-2, stem cell transplantation

\section{Summary}

Purpose. This Phase III randomized multicenter trial compared progression-free (PFS) and overall survival (OS) for autologous peripheral blood stem cell (aPBSC) transplantation with or without immunotherapy in high-risk breast cancer patients.

Methods. Eligible patients had American Joint Committee on Cancer (AJCC) 5th Edition Stage II/IIIA with $\geq 4$ axillary nodes, Stage IIIB, or chemotherapy-sensitive or stable Stage IV disease. Following treatment with cyclophosphamide, thiotepa and carboplatin (STAMP V), patients were randomized to aPBSC transplant with or without immunotherapy. Patients on immunotherapy received cells that were incubated in interleukin-2 (IL-2) for $24 \mathrm{~h}$ followed by parenteral IL-2 for 5 days then 2 days of rest for 4 weeks.

Results. Fifty-nine patients were treated (35 Stage II/IIIA; 13 Stage IIIB; 11 Stage IV), 30 patients were randomized to immunotherapy and 29 patients to no immunotherapy. Neutrophils engrafted a median of 10 days post-transplant in both groups. The median times to platelet engraftment were 9 and 10 days after transplant in the no-immunotherapy and immunotherapy groups, respectively $(p=0.03)$. There was no statistical evidence $(p=0.61)$ of a difference in progression-free and surviving (PFS) at 3 years for patients receiving immunotherapy (53\%) compared with no immunotherapy (48\%). There was some evidence of superiority in overall survival (OS) at 3 years for patients receiving immunotherapy (83\%) compared with no immunotherapy (69\%), but the difference between survival curves was not statistically significant $(p=0.08)$. Also, there was some evidence that patients developing acute graft versus host disease (aGVHD) had superior PFS $(p=0.02)$ but not OS $(p=0.19)$ than patients not developing aGVHD. Toxicities were transient and similar between groups, with no treatment-related deaths.

Conclusions. This phase III study of high-risk breast cancer patients randomized to immunotherapy or no immunotherapy demonstrated that a well-tolerated immunotherapy regimen added to aPBSC transplant did not improve PFS, but there was some improvement in OS, but not by an amount that was statistically significant $(p=0.08)$.

\section{Introduction}

The role of high dose chemotherapy with peripheral blood stem cell (PBSC) transplantation for the treatment of high-risk breast cancer remains controversial [1-8]. Although in vitro data have demonstrated enhanced cytotoxicity with increasing doses of chemotherapy, escalation of dose alone is unlikely to eliminate all malignant cells [9]. Recent clinical trials question the role of high dose chemotherapy and bone marrow transplantation and support an urgent need to develop innovative therapies [10]. Immunotherapy following myelosuppressive chemotherapy provides an attractive modality of non-cross-resistant tumor cell killing in patients with minimal residual disease. 
We previously generated activated effector cells in vitro with $24 \mathrm{~h}$ of incubation of PBSC with interleukin-2 (IL-2). In high-risk breast cancer patients, a clinical syndrome suggestive of cutaneous autologous graft versus host disease (aGVHD) associated with IL-2 activated autologous PBSC (aPBSC) transplantation followed by 4 weeks of low dose IL-2 was observed [11-13].

Because of encouraging preclinical and clinical Phase I and II trial results, a multicenter phase III randomized clinical trial was designed to compare this immunotherapy regimen to a standard transplant regimen [14], involving cyclophosphamide, thiotepa and carboplatin (STAMP V). The primary endpoint was progressionfree survival (PFS) and the major objectives were to compare the PFS and overall survival (OS) between the two treatment arms. Secondary objectives included comparison of engraftment and toxicities.

\section{Methods}

Patient population, eligibility criteria and staging evaluation

Women between the ages of 17 and 70 years with AJCC 5th Edition Stage II (T1 or T2 with $\geq 4$ axillary lymph nodes involved with disease), Stage III (T3 with involved lymph nodes or T4) or chemotherapy-sensitive or stable Stage IV disease, were eligible. Patients had to have adequate bone marrow, liver and renal function, no history of debilitating cardiac or pulmonary disease, and a Karnofsky performance status of $\geq 80 \%$. A left ventricular ejection fraction of $\geq 50 \%$ measured by radionuclide scan and pulmonary function tests demonstrating a forced expiratory volume $\left(\mathrm{FEV}_{1}\right)$ of $>70 \%$ and a diffusion capacity $>60 \%$ were needed. No radiographic evidence of malignant central nervous system involvement could exist. Negative serologies for Hepatitis B surface antigen, HIV, and HTLV I, and histological negative bone marrow aspirate and biopsy were required. The protocol received institutional review board approval and all patients signed informed consent.

The initial staging evaluation included a complete physical examination, chest radiograph, bone scan, computer tomographs of the head, thorax, abdomen, and pelvis, electrocardiogram, and audiogram. Laboratory evaluation included a complete blood count with differential and platelet count, coagulation profile, and renal, liver, and thyroid function tests. Patients with measurable disease had baseline tumor measurements by physical examination and/or radiographic studies.

\section{Peripheral blood stem cell (PBSC) collection and harvest}

Prior to priming chemotherapy, a central venous catheter was placed. Paclitaxel $\left(300 \mathrm{mg} / \mathrm{m}^{2}\right)$ was administered as an intravenous infusion over $24 \mathrm{~h}$ to mobilize PBSC (Figure 1). Recombinant human granulocyte colony stimulating factor (rhG-CSF) $(5 \mu \mathrm{g} / \mathrm{kg})$ was administered subcutaneously $48 \mathrm{~h}$ after priming chemotherapy administration and continued daily until apheresis completion. Complete blood counts were obtained every other day until the start of leukapheresis.

PBSC collection began $24 \mathrm{~h}$ after the white blood count (WBC) returned to $1 \times 10^{9} / 1$. Cells were collected using a Cobe Spectra Cell Separator (Cobe Laboratories, Lakewood, Colorado) with Anticoagulant Citrate Dextrose-formula A (ACD-A) (Baxter Healthcare, Deerfield, IL). Three to four whole blood volumes (10-20 1) were processed for each collection. Leukapheresis continued until $3 \times 10^{6} \mathrm{CD}^{+} 4^{+}$cells $/ \mathrm{kg}$ and $7.5 \times 10^{8}$ mononuclear cells $/ \mathrm{kg}$ of body weight were obtained. Clonogenic progenitor cell assays were performed for each collection. Concentrated cell suspensions were cryopreserved in medium consisting of $6 \%$ Pentastarch (McGaw, Irvine, CA), 5\% DMSO (Cryoserv, Research Industries, Salt Lake City, UT), 4\% human serum albumin (American Red Cross, Washington, DC), and either sterile endotoxin- tested bovine pancreatic DNase (Sigma Chemicals, St. Louis, MO) or recombinant human DNase (Pulmozyme, Genentech, San Francisco, CA). Cells were frozen in Cryocyte bags (Baxter Healthcare) and stored in liquid nitrogen.

\section{Interleukin-2 activation of $P B S C$}

IL-2 activation of PBSC has been described previous [15] Briefly, harvested cells were thawed rapidly in a $37{ }^{\circ} \mathrm{C}$ water bath and incubated for $24 \mathrm{~h}$. Incubation was in $5 \% \mathrm{CO}_{2}$ at $37{ }^{\circ} \mathrm{C}$ in the serum-free medium $\mathrm{X}$ VIVO 10 (BioWhittaker, Walkersville, MD) containing gentamicin (50 ug/ml), L-glutamine, heparin (50 units/ ml) (Elkins-Simm, Inc., Cherry Hill, NJ) and IL-2 (6000 IU/ml) (Chiron, Emeryville, CA). Samples were obtained from the thawed cell suspension prior to and following IL-2 activation and analyzed for cell count, viability, Gram stain, immunophenotyping, cytotoxicity assays and sterility. Approximately one third of the PBSC were stored for backup without IL-2 culturing.

\section{Treatment plan}

Cyclophosphamide $\left(1500 \mathrm{mg} / \mathrm{m}^{2}\right)$, carboplatin $(200$ $\left.\mathrm{mg} / \mathrm{m}^{2}\right)$ and Thiotepa $\left(125 \mathrm{mg} / \mathrm{m}^{2}\right)$ each were administered intravenously over $2 \mathrm{~h}$ for 4 days on days -6 to -3. MESNA (sodium 2-mercaptoethane sulfonate) and hydration were given as prophylaxis for hemorrhagic cystitis. The anti-emetic regimen included ondansetron, lorazepam, and diphenhydramine.

On day 0, the PBSC were infused over 30-90 min at the patient's bedside with cardiovascular monitoring. Patients randomized to no immunotherapy received no further treatment. Patients randomized to immunotherapy received subcutaneous IL-2 (Chiron Therapeutics, California) the same day and continued 


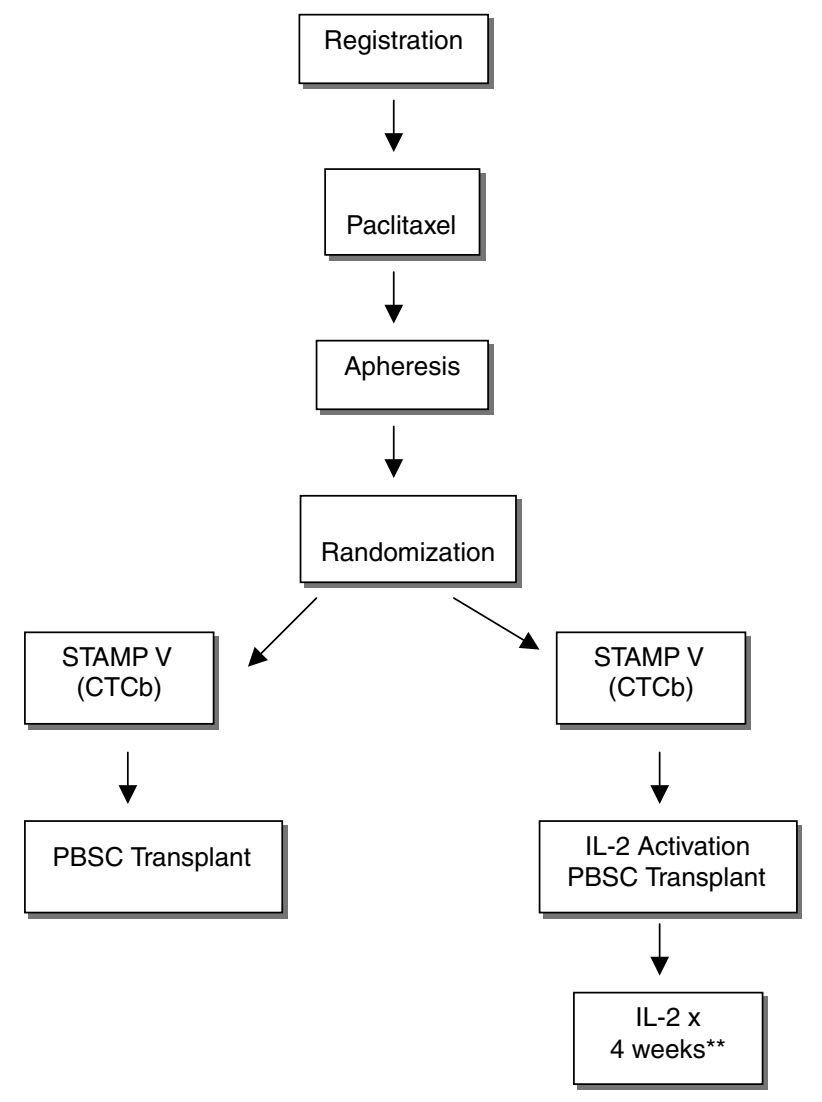

Abbreviations: $\mathrm{CTCb}=$ cyclophosphamide, Thiotepa, carboplatin PBSC $=$ peripheral blood stem cells

** Stage IV patients will receive IL-2 at 1.8 × 106 I.U./m2/d

5 days every month until progression

Figure 1. Trial Schema. 30 Patients were randomized to immunotherapy and 29 patients to no immunotherapy. All randomized patients received transplant.

for 4 weeks [11] IL-2 began at $6 \times 10^{5} \mathrm{IU} / \mathrm{m}^{2} / \mathrm{d}$, based on previous clinical trials $[11,13]$. A week of therapy was 5 days of treatment and 2 days of rest. Engraftment was defined as an absolute neutrophil count of $\geq 500 / \mathrm{mm}^{3}$ for 3 days and a platelet count of $\geq 20 \times 10^{9} / 1$ for 3 days (untransfused).

Toxicities were monitored daily and graded according to CALGB Toxicity Criteria as follows: Grade 0 (no toxicity), Grade 1 (mild), Grades 2 and 3 (moderate), Grade 4 (severe) and Grade 5 (toxic death). IL-2 was held if severe non-hematologic toxicities occurred during therapy. If the toxicities returned to Grade 1 or resolved within 48 h, IL-2 was resumed. If signs and symptoms of the non-hematologic toxicities returned after resolution or failed to improve in $72 \mathrm{~h}$, the IL-2 was discontinued but the patient remained on study.

\section{Evaluation of skin for GVHD}

Since prior clinical trials using this immunotherapy regimen demonstrated signs consistent with skin aGVHD, each patient received a daily skin exam by a bone marrow transplant physician during their in-hospital stay. Individuals randomized to immunotherapy treatment at Georgetown University Medical Center (GUMC) additionally were to receive a $4-\mathrm{mm}$ skin punch biopsy prior to initiation of week 3 therapy. All biopsy specimens were graded for the presence or absence of 4 histologic criteria using a scale developed by Horn [16] including basal cell vacuolization, exocytosis, dyskeratotic cells and dermal lymphatic infiltration by a dermatopathologist blinded to the treatment the patient was receiving. Skin biopsy specimens demonstrating $\geq 3$ criteria were considered compatible with aGVHD.

\section{Supportive care}

Patients were treated in HEPA-filtered rooms. Routine supportive care was started at the time of admission and included Norfloxacin for gastrointestinal bacterial decontamination, Fluconazole, and Acyclovir (both continued until day 30). With the development of a temperature $\geq 38.5^{\circ} \mathrm{C}$, broad-spectrum antibiotics were initiated and Norfloxacin was discontinued. Amphotericin B was administered empirically when patients remained febrile after 48-72 h on broad-spectrum antibiotic therapy. All patients received daily rhG-CSF $(5 \mu \mathrm{g} / \mathrm{kg} /$ day) beginning on day 5 after transplantation until the absolute neutrophil count (ANC) reached $5.0 \times 10^{9} / 1$ for 2 days.

\section{In-hospital clinical monitoring}

Patients underwent daily physical examination and blood work, including a complete blood count with differential and platelet count, electrolytes, and liver and renal function tests. Packed red blood cells and platelets were administered if the hemoglobin level fell below $8.5 \mathrm{~g} / \mathrm{dl}$, platelets dropped below $20 \times 10^{9} / 1$, or for symptomatic anemia or bleeding.

\section{Clinical monitoring after discharge}

Patients were evaluated weekly in the outpatient clinic for 1 month before returning to their referring oncologist. Evaluation at each clinic visit included a history and physical examination, a complete blood count with differential and platelet count, electrolytes, liver and renal function tests. Response was evaluated 100 days after transplantation by physical examination, bone scan and $\mathrm{CT}$ scans of the thorax, abdomen and pelvis. Re-evaluation was performed every 6 months for the first 2 years and yearly thereafter. Patients with inflammatory breast cancer or 4 or more axillary lymph nodes involved with metastatic carcinoma received irradiation of the ipsilateral chest wall, supraclavicular, and axillary areas beginning 3 months post-transplantation (total dose $5040 \mathrm{cGy}$ chest wall). Patients with estrogen receptor (ER) positive tumors started on Tamoxifen (20 mg twice a day) after completion of chest wall irradiation. 


\section{Statistical considerations}

Design of the study

This randomized, phase III clinical trial was stratified by disease stage (II-IIIA, IIIB, and IV) and conducted at multiple institutions. The trial was designed to determine whether patients receiving additional immunotherapy with subcutaneous IL-2 cells (immunotherapy group) would demonstrate improved PFS at 3 years compared with patients receiving combined therapy and transplant with PBSCs alone (no-immunotherapy group). Originally, 195 patients were to be accrued and followed for 3 years to detect an odds ratio (OR) for PFS of 2.0 or more over all strata with statistical power of $80 \%$ at a $5 \%$ significance level (two-sided test). In stratum Stage II-IIIA, an OR of 2.0 would mean an improvement in 3-year PFS from $60 \%$ for the no-immunotherapy group to $75 \%$ for the immunotherapy group, and there would be similar amounts of improvement in PFS in the other stages. Secondary endpoints included OS, hematopoietic reconstitution, and toxicity.

\section{Conduct of the study}

Between December 1997 and December 2000, 59 patients were accrued by Georgetown University Medical Center (GUMC) $(n=45)$, University of Massachusetts Memorial Hospital $(n=8)$ and Holy Cross Hospital $(n=6)$. Patients were stratified by stage of disease and randomized equally to the two treatment arms. A biostatistician prepared the randomization list that was concealed from other study personnel. At completion of mobilization treatment, the research nurse provided the patient's disease stage to the biostatistician for randomization. Study personnel and patients were unblinded to treatment after randomization since different procedures were required for immunotherapy treatment. The dermatopathologist was blinded to treatment arm (immunotherapy versus not) for aGVHD ascertainment.

In accord with NCI requirements for phase III clinical trials, an independent Data Monitoring Committee (DMC) was organized, that included one biostatistician and two clinicians. The DMC met in January 1999 and December 2000 and recommended termination of the study following the latter meeting. The major reason for termination was a marked drop in patient accrual following publications questioning the role of transplantation for breast cancer $[1,6,17]$.

\section{Analysis of the study}

PFS, OS, and post-progression survival (PPS) curves were calculated using the Kaplan-Meier [18] method. PFS was defined as the time from randomization to progression, death, or date of last follow-up, whichever came first. Overall survival was the time between randomization and date of death or last contact. Patients surviving progression-free were censored at the date of last contact for PFS. PPS, the time between progression and date of death or last follow-up, included only pa- tients who progressed. Survival curves for all patients were compared by the generalized Wilcoxon test [19] since hazard rates were not proportional between groups. Survival curves were also calculated for subsets of patients by stage and by aGVHD without adjustment of $p$-values since interpretation of outcomes was based upon patients from all stages combined. The combination of treatment and aGVHD effects on PFS and OS was explored with Cox's proportional hazards regression [20]. Standard $\chi^{2}$ tests were used to compare the distributions of patients and incidence rates (e.g. toxicity) between groups. All statistical tests were two-sided with a 0.05 significance level. Analyses were performed using the 'intention-to-treat' principle. The HaybittlePeto approach to the analysis of accumulating data was planned with interim tests to be performed at a 0.001 significance level, assuring that the final test would be nearly at the 0.05 level. No interim analyses of efficacy were performed prior to termination of the study.

\section{Results}

\section{Patient characteristics}

Fifty-nine patients with a median age of 49 years (range 32-66 years) were treated. Thirty patients were randomized to receive immunotherapy and the remaining 29 patients to receive standard aPBSC transplant therapy. Table 1 presents patient demographics and the comparability of the two treatment groups. In addition to stage which was a stratification factor, patients had comparable distributions of age group, ER and PR status, and race.

\section{Engraftment}

All patients engrafted, so backup stem cell infusion was not needed. The median number of days required for the absolute neutrophil count (ANC) to maintain $500 / \mathrm{mm}^{3}$ for 32 days was 10 days in both treatment groups (range 8-12 days). Platelet engraftment occurred at median of 9 days post-transplant (range 6-22 days) for the noimmunotherapy arm and day 10 (range 6-25 days) in the immunotherapy arm $(p=0.03)$.

\section{Withdrawal from immune therapy}

Six of the 30 patients randomized to receive immunotherapy were removed from IL-2 due either to infection $(n=5)$ or patient's desire $(n=1)$. The median duration of IL-2 therapy for these six patients was 14 days (range: 4-21 days).

\section{Progression-free Survival, overall survival, and post-progression survival}

The median follow-up time for surviving patients was 56 months. Figure $2 \mathrm{a}$ gives the PFS curves by 
Table 1. Comparability of patients by treatment Arm

\begin{tabular}{|c|c|c|c|c|}
\hline \multirow[t]{2}{*}{ Patient Characteristics } & \multicolumn{4}{|l|}{ Treatment } \\
\hline & All N (\%) & Immunotherapy $\mathrm{N}(\%)$ & No Immunotherapy N (\%) & $p$-value* \\
\hline All Patients & $59(100)$ & $30(51)$ & $29(49)$ & \\
\hline \multicolumn{5}{|l|}{ Stage } \\
\hline II-IIIA & 35 (59) & $18(60)$ & $17(59)$ & \\
\hline IIIB & $13(22)$ & $6(20)$ & $7(24)$ & \\
\hline IV & $11(19)$ & $6(20)$ & $5(17)$ & \\
\hline Age (Yrs.) & & & & $>0.99$ \\
\hline $30-39$ & $10(17)$ & $5(17)$ & $5(17)$ & \\
\hline $40-49$ & $24(41)$ & $12(40)$ & $12(41)$ & \\
\hline $50-59$ & $20(34)$ & $10(33)$ & $10(34)$ & \\
\hline $60-69$ & $5(8)$ & $3(10)$ & $2(7)$ & \\
\hline Treatment Site & & & & 0.18 \\
\hline LCC & $45(76)$ & $24(80)$ & $21(72)$ & \\
\hline $\mathrm{HCH}$ & $6(10)$ & $1(3)$ & $5(17)$ & \\
\hline UMA & $8(14)$ & $5(17)$ & $3(10)$ & \\
\hline ER Status & & & & 0.20 \\
\hline+ & $29(49)$ & $15(50)$ & $14(48)$ & \\
\hline- & $24(41)$ & $14(47)$ & $10(34)$ & \\
\hline UNK & $6(10)$ & $1(3)$ & $5(17)$ & \\
\hline PR Status & & & & 0.21 \\
\hline+ & $27(46)$ & $14(47)$ & $13(45)$ & \\
\hline- & $26(44)$ & $15(50)$ & $11(38)$ & \\
\hline UNK & $6(10)$ & $1(3)$ & $5(17)$ & \\
\hline Race & & & & 0.17 \\
\hline African-American & $7(12)$ & $2(7)$ & $5(17)$ & \\
\hline Asian & $2(4)$ & $0(0)$ & $2(7)$ & \\
\hline Caucasian & $48(81)$ & $26(87)$ & $22(76)$ & \\
\hline Hispanic & $1(2)$ & $1(3)$ & $0(0)$ & \\
\hline Other & $1(2)$ & $1(3)$ & $0(0)$ & \\
\hline
\end{tabular}

LCCC = Lombardi Comprehensive Cancer Center, Georgetown University Medical Center; HCH = Holy Cross Memorial Hospital; UMA $=$ University of Massachusetts Memorial Medical Center; ER = Estrogen Receptor; PR = Progesterone Receptor; UNK = Unknown or Missing.

*The $p$-value is provided to indicate a level of difference between the two groups. However, the interpretation is not based on determining if these differences occurred at random since the study was randomized, so all differences occurred at random.

treatment. Although the PFS curves appeared to separate during the first 18 months, they converged by 24 months and there was no statistical evidence of differing PFS times between groups $(p=0.61)$. The 3 -year PFS was $53 \%(\mathrm{SE}=9 \%)$ in the immunotherapy group and $48 \%(\mathrm{SE}=9 \%)$ in the no-immunotherapy group.

Figure $2 \mathrm{~b}$ gives the OS curves by treatment group and, subsequent to 1 year, the immunotherapy group had superior survival. At 3 years, the OS was $83 \%$ $(\mathrm{SE}=7 \%)$, in the immunotherapy group and $69 \%$ $(\mathrm{SE}=9 \%)$ in no-immunotherapy group. The apparent visual advantage in OS for the immunotherapy group did not achieve statistical significance at the 5\% level $(p=0.08)$. Of the 23 deaths on the study, 14 were in the no-immunotherapy group.

Figure 2c gives the PPS curves for progressed patients. The median post-progression follow-up time among surviving patients was 18 months. The 18-month survival post-progression was $62 \%(\mathrm{SE}=12 \%)$ in the immunotherapy group and $44 \%(\mathrm{SE}=13 \%)$ in the noimmunotherapy group. The difference between curves was not significant $(p=0.13)$, and the $p$-value was identical to that in Figure $2 b$ for the OS curves. The apparent difference in OS experience from the time of randomization between the immunotherapy and no immunotherapy arms was due primarily to longer survival post-progression in the immunotherapy group.

\section{Progression-free survival and overall survival based on disease stages}

Figure $3 \mathrm{a}$ and $3 \mathrm{~b}$ provide the PFS and OS experience by treatment for the 35 Stage II-IIIA patients, the largest subgroup. While the initial trial design included a stratification by disease stage, the actual number of patients in each disease stage was quite small given the early termination of the study secondary to poor accrual to transplant trials. In Figure $3 \mathrm{a}$, the estimated PFS at 


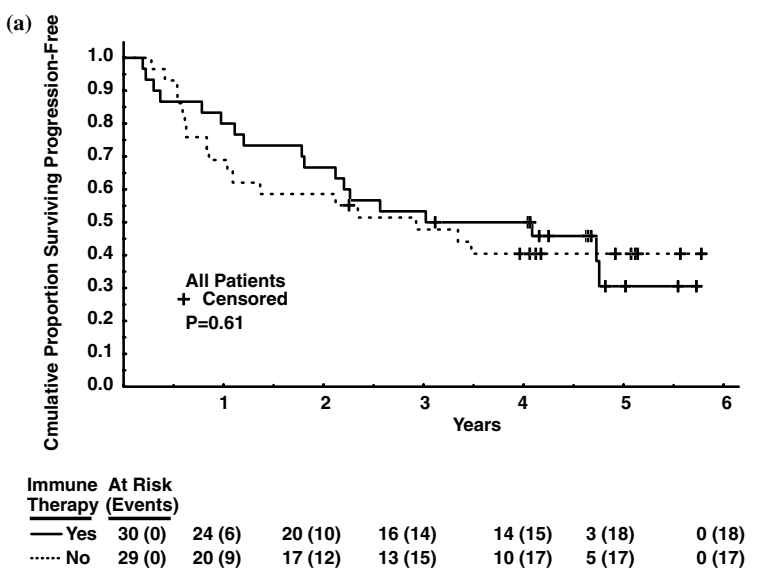

(b)

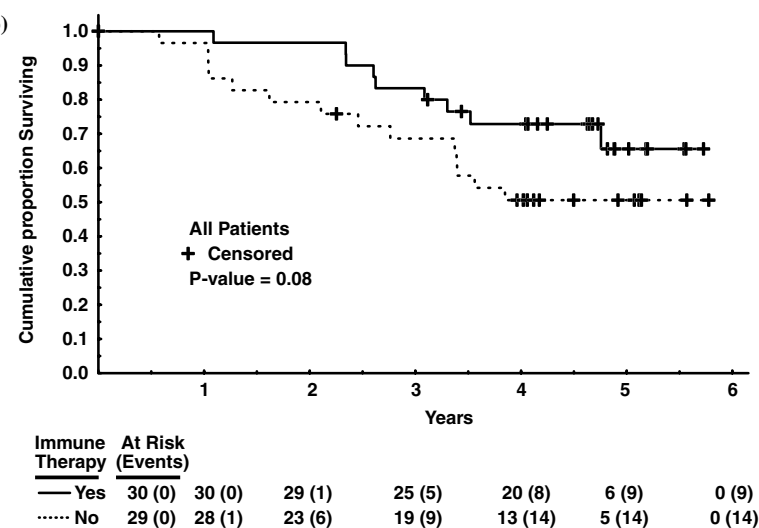

(1)

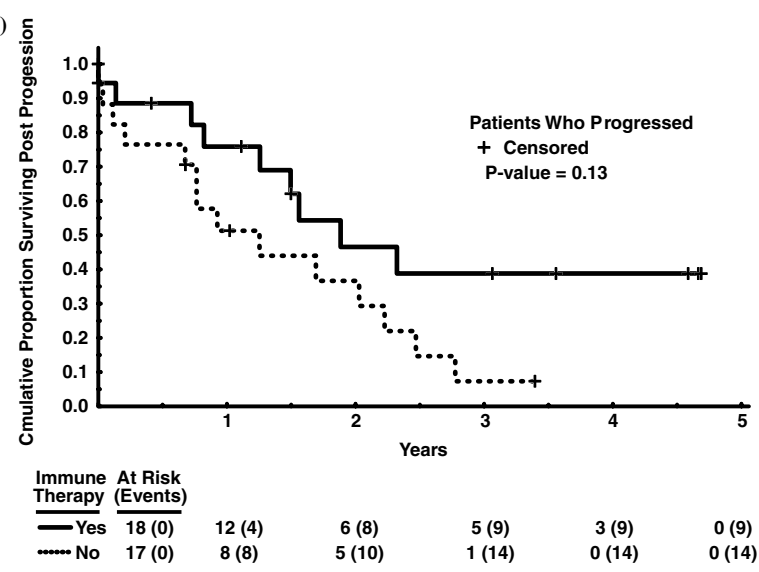

Figure 2. All patients. Progression-free (a), overall (b), and post progression (c) survival for the immunotherapy (solid), and no-immunotherapy (dashed) groups.

3 years in the immunotherapy group was $61 \%$ $(\mathrm{SE}=11 \%)$ compared with $46 \%(\mathrm{SE}=12 \%)$ in the no-immunotherapy group, but the difference between curves was not statistically significant $(p=0.34)$. Figure $3 \mathrm{~b}$ gives the survival curves by treatment in Stage II-IIIA patients, showing some advantage in survival for patients receiving immunotherapy $(p=0.05)$. At 3 years, $83 \%$ ( $\mathrm{SE}=9 \%)$ of patients were surviving in the immunotherapy group compared with $64 \%$ (SE $=12 \%)$ in the no-immunotherapy group.

The PFS at 3 years for the 13 stage IIIB patients was $50 \%(\mathrm{SE}=20 \%)$ in the immunotherapy group and $43 \%(\mathrm{SE}=19 \%)$ in the no-immunotherapy group
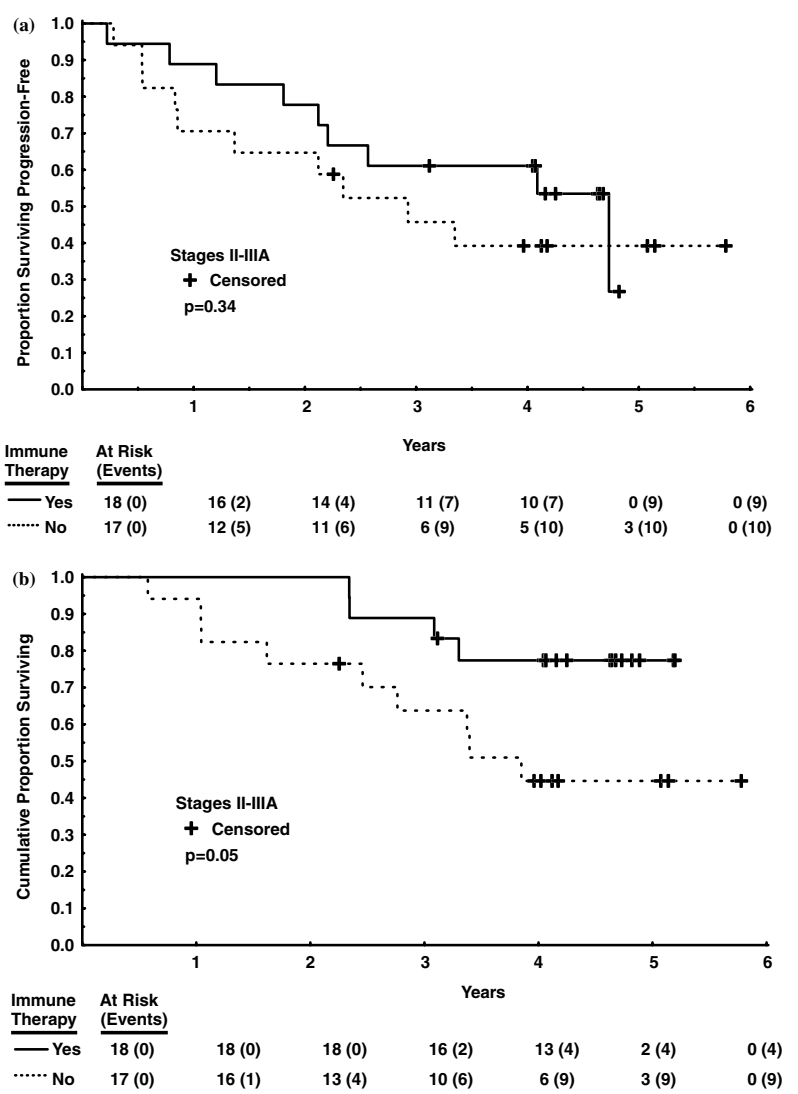

Figure 3. Stages II-IIIA. Progression-free (a) and overall (b) survival for patients with stages II-IIIA in the immunotherapy (solid), and noimmunotherapy (dashed) groups.

$(p=0.84)$. The percent of stage IIIB patients surviving at 3 years was $83 \%(\mathrm{SE}=15 \%)$ in the immunotherapy group and $86 \%(\mathrm{SE}=13 \%)$ in the no-immunotherapy group ( $p=0.91$; curves not shown).

There were only 11 patients with Stage IV disease in the study. Patients with Stage IV disease receiving immunotherapy had $33 \%$ PFS at 2 years $(33 \%$; SE $=19 \%)$ compared to $60 \%(\mathrm{SE}=22 \%)$ in the noimmunotherapy arm, but the difference between curves was not significant $(p=0.38)$. The OS curves were similar for the immunotherapy and no-immunotherapy groups, with 3-year estimates of the percent surviving of $63 \%(\mathrm{SE}=21 \%)$ and $60 \%(\mathrm{SE}=22 \%)$, respectively $(p=0.87$; curves not shown).

\section{Evaluation of cutaneous aGVHD}

Of the 45 patients treated at GUMC, 33 received $4 \mathrm{~mm}$ punch skin biopsies upon engraftment, 16 (48\%) who were classified as having aGVHD and 17 patients without. There was a higher percent of patients with aGVHD in the no-immunotherapy arm $(67 \% ; 8 / 12)$ compared to the immunotherapy arm $(38 \% ; 8 / 21)$, but this difference was not significant $(p=0.16$ ). Figure $4 a$ shows a statistically significant advantage in PFS for patients with aGVHD $(p=0.02)$. The 3 -year PFS was $75 \%(\mathrm{SE}=11 \%)$ for the aGVHD patients compared with $29 \%(\mathrm{SE}=11 \%)$ for the patients without 

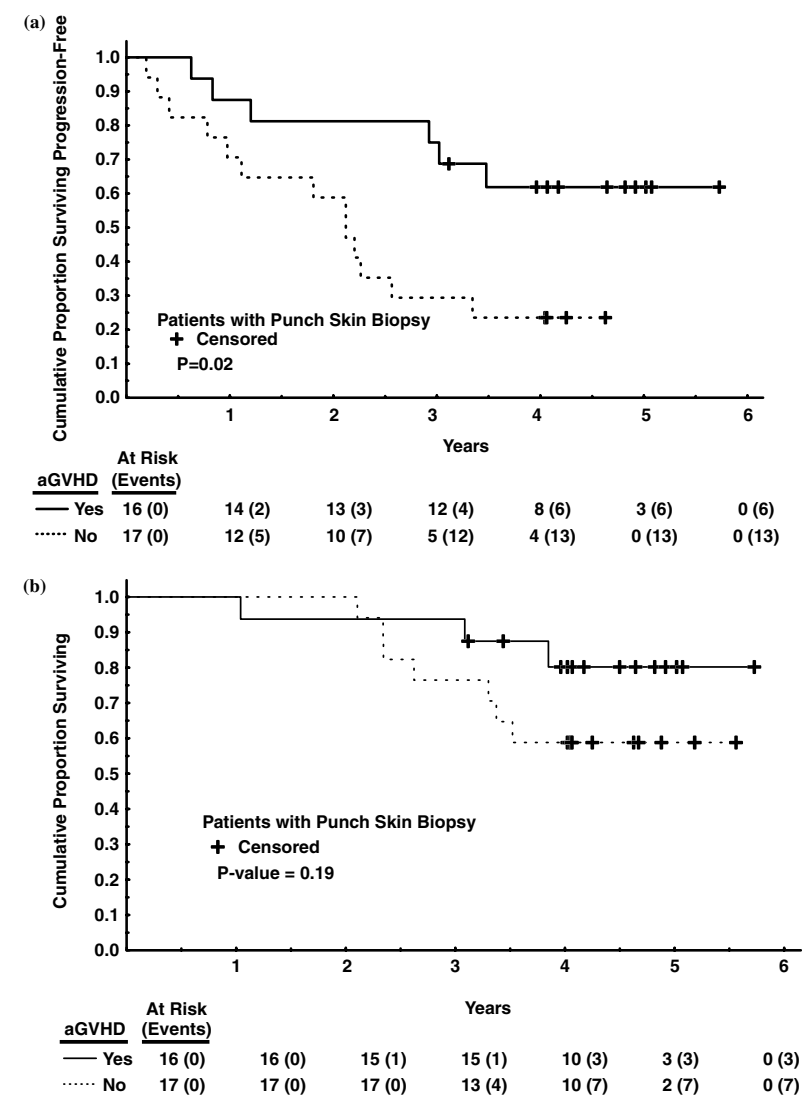

Figure 4. aGVHD. Progression-free (a) and overall (b) survival for patients with punch biopsies with (solid), and without (dashed) aGVHD.

aGVHD $(p=0.02)$. Survival curves for patients who had or did not have aGVHD are shown in Figure 4b. There was no statistically significant difference between the curves $(p=0.19)$, the percent of patients surviving 3 years being $94 \%(\mathrm{SE}=6 \%$ ) for the aGVHD patients and $76 \%(\mathrm{SE}=10 \%)$ for patients not having aGVHD.

Cox regression models were fit to both the PFS and OS data including factors for treatment and occurrence of aGVHD (no or yes). In the model for PFS, the type of treatment was not statistically significant $(p=0.67)$, however occurrence of aGVHD was statistically significant $(p=0.02)$. Neither treatment nor aGVHD status was significantly associated with OS in the Cox model. With only 33 patients that were classified for aGVHD, additional studies are needed to confirm the prognostic value of aGVHD.

\section{The incidence of in-hospital moderate (grade 3 ) or severe toxicities (grade 4)}

There were no toxic deaths and all side effects were self-limiting. Toxicities are presented by treatment arm with severity in Table 2 . There was no statistical difference in incidence of grades 3 and 4 toxicities between the two groups. No grade 4 non-hematologic toxicities occurred in the immunotherapy arm. Three patients experienced Grade 4 toxicities in the noimmunotherapy arm, consisting of diarrhea, dysphagia, stomatitis, or skin rash.

\section{Cause of death}

With the median follow-up time among surviving patients of 56 months, a total of 23 patients died, 9

Table 2. Numbers (and percentages) of patients experiencing moderate (grade 3) or severe (grade 4) toxicities

\begin{tabular}{|c|c|c|c|c|}
\hline \multirow[t]{4}{*}{ Toxicity } & \multicolumn{2}{|c|}{ Immunotherapy $\mathrm{N}=30$} & \multicolumn{2}{|c|}{ No Immunotherapy $\mathrm{N}=29$} \\
\hline & \multicolumn{2}{|l|}{ Grade } & \multicolumn{2}{|l|}{ Grade } \\
\hline & 3 & 4 & 3 & 4 \\
\hline & $\mathrm{N}(\%)$ & $\mathrm{N}(\%)$ & $\mathrm{N}(\%)$ & $\mathrm{N}(\%)$ \\
\hline Cardiac & $1(3)$ & $0(0)$ & $1(3)$ & $0(0)$ \\
\hline Esophagitis/stomatitis & $2(7)$ & $0(0)$ & $2(7)$ & $2 *(7)$ \\
\hline Flu-like symptoms & $4(14)$ & $0(0)$ & $2(7)$ & $0(0)$ \\
\hline GI symptoms & $2(7)$ & $0(0)$ & $3 * *(10)$ & $1(3)$ \\
\hline Infection & $3(10)$ & $0(0)$ & $4(14)$ & $0(0)$ \\
\hline Liver transaminase & $1(3)$ & $0(0)$ & $0(0)$ & $0(0)$ \\
\hline Metabolic abnormalities & $4(14)$ & $0(0)$ & $3(10)$ & $0(0)$ \\
\hline Coagulation & $0(0)$ & $0(0)$ & $0(0)$ & $1(3)$ \\
\hline Phlebitis/ thrombosis Embolism & $0(0)$ & $0(0)$ & $1(3)$ & $0(0)$ \\
\hline Pulmonary & $1(3)$ & $0(0)$ & $1(3)$ & $0(0)$ \\
\hline Skin & $1(3)$ & $0(0)$ & $1(3)$ & $1(3)$ \\
\hline
\end{tabular}

Metabolic abnormalities = Hyperglycemia, hypocalcemia, hypomagnasemia, or hyponatremia. Flu-like symptoms $=$ Fever without infection, Malaise/fatigue, or pain. GI symptoms = Anorexia, diarrhea, or nausea

*One patient had grade 4 toxicities for both esophagitis and stomatitis; she is counted only once here, but there are a total of 3 grade four toxicities in this category.

**Two patients each had grade 3 toxicities for both anorexia and nausea. Each patient is only counted once here, but there are a total of 5 grade three toxicities in this category. Grade $4=$ severe/life-threatening toxicity. 
patients randomized to immunotherapy and 14 patients who were not. Of the patients expiring on immunotherapy, 7 patients died of tumor and 2 patients had unknown cause of death. Of the 14 patients not receiving immunotherapy, 13 died a known tumor-related death.

\section{Discussion}

The regimen using IL-2 activation of PBSCs and IL-2 following transplantation demonstrated some improvement in OS compared to standard aPBSC therapy in these high risk breast cancer patients that was not statistically significant $(p=0.08)$. This trial was planned to accrue 195 patients in order to detect a significant difference between the treatment arms (odds ratio for PFS of 2.0 or greater with statistical power of $80 \%$ at a $5 \%$ significance level). Due to the marked drop in enthusiasm for high dose chemotherapy and stem cell transplant in patients with breast cancer, only 59 patients were accrued before the study termination was recommended by the independent data safety monitoring board. Thus, any conclusions reached from this trial are underpowered, and must be viewed as tentative. There was no difference in PFS between the treatment groups so the difference in OS was due primarily to patients who received IL-2 living longer post-progression than patients without IL-2. For skin biopsy patients, aGVHD was associated with longer PFS $(p=0.02)$, but not OS $(p=0.19)$. Toxicities, aGVHD, and neutrophil engraftment were similar between arms. Platelet engraftment was delayed one day in the immunotherapy arm.

Immunotherapy following aPBSC transplantation has been shown to generate aGVHD in patients with breast cancer, multiple myeloma, lymphoma and acute myelogenous leukemia [11,13,21-28]. In the allogeneic bone marrow transplant setting, the presence of GVHD is associated with a GVT effect, possibly contributing to lower relapse rates $[29,30]$. The co-existence of an autologous GVT effect among patients experiencing aGVHD remains unknown.

The mechanism of action for the immunotherapy in this protocol differs from other regimens. Researchers at Johns Hopkins have used cyclosporin and $\gamma$-IFN posttransplant. It is postulated that the $\gamma$-IFN upregulates MHC Class II expression, while the cyclosporin prevents the deletion of auto-reactive lymphocytes. As a result, the auto-reactive lymphocytes recognize and attack the patient's cells with the enhanced MHC Class II expression.

Incubation of hematopoietic cells with IL-2 in vitro for $24 \mathrm{~h}$ generates cytotoxic effector cells [21,22,26,31]. These activated cells lyse NK-sensitive and NK-resistant tumor cells and result in in vitro purging [32,33]. Additionally, infusion of IL-2 activated aPBSC followed by low doses of parenteral IL-2 reduces tumor cell contamination within the graft and generates cytotoxic effector cells that may mediate a GVT effect in vivo [34-36].

Prior to implementing IL-2 activation of stem cells in clinical trials, growth and differentiation of hematopoietic progenitor cells had to remain unaffected. When bone marrow or peripheral blood stem cells are activated in IL-2 in long-term (7 days) and short-term (1 days) culture, there is no decrease in colony-forming cells, when analyzed by clonogenic assays [15,36]. A previous Phase I-II Clinical Trial with 61 patients demonstrated that the in vitro activation of aPBSC with IL-2 for transplantation followed by the same day parenteral administration of IL-2 did not delay engraftment and was associated with mild to moderate toxicities in Stage II-IV breast cancer patients [13]. In addition, some of these patients demonstrated signs and symptoms of cutaneous aGVHD [12]. The IL-2 activated bone marrow has significant anti-tumor activity in vitro and in vivo with no detrimental effect on hematopoiesis [35]. Previous breast cancer clinical trials using IL-2 activation of PBSC combined with parenteral IL-2 or the combination of IL- 2 with $\alpha$-IFN post-transplant, revealed no detrimental effect on hematopoiesis or engraftment [12,37].

Forty-eight hours or more of IL-2 incubation of peripheral blood mononuclear cells is required to generate lymphokine activated killer (LAK) cells [15,26,38]. This clinical trial utilized $24 \mathrm{~h}$ of IL-2 activation of PBSC to induce cytotoxic effector cells, suggesting that different cells may be involved. Although others have demonstrated the importance of $\mathrm{T}$ cell subsets in shortterm IL-2 activation, especially $\mathrm{CD}^{+}$and $\mathrm{CD}^{+}{ }^{+} \mathrm{T}$ cells [38], our results demonstrate that the majority of effector cells generated with 24-h IL-2 incubation are $\mathrm{CD}^{+} \mathrm{T}$ cells, $\mathrm{CD}^{+} 6^{+} \mathrm{NK}$ cells, and possibly, $\mathrm{CD}^{+} \mathrm{CD}^{+} 6^{+}$cells [39]. Recent evidence shows that 5-30\% of $\mathrm{CD}^{+} \mathrm{T}$ cells co-express CD56 NK marker [40]. The cytokine-induced killer (CIK) cell, a cell possessing the combination of $\mathrm{T}$ cell markers $\left(\mathrm{CD}^{+}\right)$and NK cell marker $\left(\mathrm{CD} 56^{+}\right)$may also play a role, since the $\mathrm{CD}^{+} \mathrm{CD}^{+} 6^{+}$cells also contributed to cytotoxicity $[41,42]$

The role of aPBSC transplantation for high-risk breast cancer patients has not yet been clearly defined [43]. Because of publications questioning the role of transplantation for breast cancer, accrual to this trial dropped markedly, causing the investigators to terminate patient accrual early, as recommended by the DMC. Although patients receiving immunotherapy experienced improved OS with median follow-up of 56 months in surviving patients, especially patients with Stage II-IIIA disease, the number of patients is small and there was no evidence of an improvement in PFS. These intriguing results from combining immunotherapy with myelosuppressive chemotherapy should be considered in the design of future clinical trials for high-risk breast cancer patients. Additionally, the relationship of outcome with aGVHD deserves further exploration. 


\section{Acknowledgement}

We thank Dr Kenneth Meehan, now at the Dartmouth-Hitchcock Medical Center, for his contributions to the design and conduct of this study while at the Lombardi Comprehensive Cancer Center of the Georgetown University Medical Center. This project was sponsored by the Lombardi Comprehensive Cancer Center SPORE Grant (P50CA58185) in Breast Cancer and the Cancer Center Support Grant (5P30CA51008).

\section{References}

1. Stadtmauer EA, O’Neill A, Goldstein LJ, Crilley PA, Mangan KF, Ingle JN, Brodsky I, Martino S, Lazarus HM, Erban JK Sickles C, Glick JH: Conventional-dose chemotherapy compared with high-dose chemotherapy plus autologous hematopoietic stemcell transplantation for metastatic breast cancer. Philadelphia bone marrow transplant group. N Engl J Med 342: 1069-1076, 2000

2. Rodenhuis S, Bontenbal M, Beex LVAM, Wagstaff J, Richel DJ, Nooij MA, Voest EE, Hupperets P, van Tinteren H, Peterse HL, TenVergert EM, deVries EGE, The Netherlands working party on Autologous Transplantation in Solid Tumors : High-dose chemotherapy with hematopoietic stem-cell rescue for high risk breast cancer. Engl J Med 349: 7-16, 2003

3. Roche H, Viens P, Biron P, Lotz JP, Asselain B: High-dose chemotherapy for breast cancer: the French PEGASE experience. Cancer Control 10: 42-47, 2003

4. Tallman MS, Gray R, Robert NJ, LeMaistre CF, Osborne CK, Vaughan WP, Gradishar WJ, Pisansky TM, Fetting J, Paietta E, Lazarus HM: Conventional adjuvant chemotherapy with or without high-dose chemotherapy and autologous stem-cell transplantation in high-risk breast cancer. N Engl J Med 349: 17-26, 2003

5. Zander AR, Kroger N, Schmoor C, Kruger W, Mobus V, Frickhofen N, Metzner B, Schultze W, Berdel WE, Koenigsmann M, Thiel E, Wandt H, Possinger K, Trumper L, Kreienberg R, Carstensen M, Schmidt EH, Janicke F, Schumacher M, Jonat W: High-dose chemotherapy with autologous hematopoietic stem-cell support compared with standard-dose chemotherapy in breast cancer patients with 10 or more positive lymph nodes: first results of a randomized trial. J Clin Oncol 22: 2273-2283, 2004

6. Antman KH: Overview of the six available randomized trials of high-dose chemotherapy with blood or marrow transplant in breast cancer. J Natl Cancer Inst Monogr : 114-116, 2001

7. The Scandinavian Breast Cancer Study Group: Results from a randomized adjuvant breast cancer study with high dose chemotherapy with $\mathrm{CTCb}$ supported by autologous bone marrow stem cells versus dose escalated and tailored FEC therapy. Proc Am Assoc Clin Oncol 18: 2a, 1999

8. Peters WP, Rosner G, Vredenburgh J, Shpall E, Crump M, Marks L, Cirrincione C, Hurd D, Norton L: Updated results of a prospective randomized comparison of two doses of combination alkylating agents as consolidation after CAF in high risk primary breast cancer involving ten or more axillary lymph nodes: CALGB 9082/SWOG 9114/NCIC MA-13. Proc Am Assoc Clin Oncol. 20: 21a, 2001

9. Peters WP, Ross M, Vredenburgh JJ, Meisenberg B, Marks B, Marks LB, Winder E, Kurtzberg J, Bast RC Jr, Jones R, Shpall E, Wu K, Rosner G, Gilbert C, Mathias B, Coniglio D, Petros W, Henderson IC, Norton L, Weiss RB, Budman D, Hurd D: Highdose chemotherapy and autologous bone marrow support as consolidation after standard-dose adjuvant therapy for high-risk primary breast cancer. J Clin Oncol 11: 1132-1143, 1993
10. Rahman Z, Frye D, Buzdar A, Smith T, Asmar L, Champlin R, Hortobagyi G: Impact of selection process on response rate and long-term survival of potential high-dose chemotherapy candidates treated with standard-dose doxorubicin-containing chemotherapy in patients with metastatic breast cancer. J Clin Oncol 15: 31713177, 1997

11. Meehan KR, Verma UN, Cahill R, Frankel S, Areman EM Sacher RA, Foelber R, Rajagopal C, Gehan EA, Lippman ME, Mazumder A: Interleukin-2-activated hematopoietic stem cell transplantation for breast cancer: investigation of dose level with clinical correlates. Bone Marrow Transplant 20: 643-651, 1997

12. Meehan KR, Verma UN, Rajogopal C, Cahill R, Frankel S, Mazumder A: Stem cell transplantation with chemoradiotherapy myeloablation and interleukin-2. J Infus Chemother 6: 28-32, 1996

13. Meehan KR, Arun B, Gehan EA, Berberian B, Sulica V, Areman EM, Mazumder A, Lippman ME: Immunotherapy with interleukin-2 and alpha-interferon after IL-2-activated hematopoietic stem cell transplantation for breast cancer. Bone Marrow Transplant 23: 667-673, 1999

14. Antman KH, Rowlings PA, Vaughan WP, Pelz CJ, Fay JW, Fields KK, Freytes CO, Gale RP, Hillner BE, Holland HK, Kennedy MJ, Klein JP, Lazarus HM, McCarthy PL, Jr., Saez R, Spitzer G, Stadtmauer EA, Williams SF, Wolff S, Sobocinski KA, Armitage JO, Horowitz MM: High-dose chemotherapy with autologous hematopoietic stem-cell support for breast cancer in North America [see comments]. J Clin Oncol 15: 1870-1879, 1997

15. Verma UN, Areman E, Dickerson SA, Kotula PL, Sacher R, Mazumder A: Interleukin-2 activation of chemotherapy and growth factor-mobilized peripheral blood stem cells for generation of cytotoxic effectors. Bone Marrow Transplant 15: 199-206, 1995

16. Horn TD: Acute cutaneous eruptions after marrow ablation: roses by other names?. J Cutan Pathol 21: 385-392, 1994

17. Rodenhuis S, van der E Richel d Wall, Schornagel J, Baars J, Koning C, Peterse J, Borger J, Nooijen W, Bakx R, Dalesio O, Rutgers E: Randomised trial of high-dose chemotherapy and haemopoietic progenitor-cell support in operable breast cancer with extensive axillary lymph-node involvement. Lancet 352: 515521, 1998

18. Kaplan E, Meier P: Nonparametric estimation from incomplete observations. J Am Stat Assoc 53: 457-481, 1958

19. Gehan E: A generalized Wilcoxon test for comparing arbitrarily singly-censored samples. Biometrika 52: 203-223, 1965

20. Cox DR : Regression models and life tables. J Roy Stat Soc B 34: 187-202, 1972

21. Charak BS, Malloy B, Agah R, Mazumder A: A novel approach to purging of leukemia by activation of bone marrow with interleukin 2. Bone Marrow Transplant 6: 193-198, 1990

22. Kennedy MJ: Induced autologous graft-versus-host disease for the treatment of cancer. Cancer Treat Rev 20: 97-103, 1994

23. Yeager AM, Vogelsang GB, Jones RJ, Farmer ER, Altomonte V, Hess AD, Santos GW: Induction of cutaneous graft-versus-host disease by administration of cyclosporine to patients undergoing autologous bone marrow transplantation for acute myeloid leukemia. Blood 79: 3031-3035, 1992

24. Jones RJ, Vogelsang GB, Hess AD, Farmer ER, Mann RB, Geller RB, Piantadosi S, Santos GW: Induction of graft-versus-host disease after autologous bone marrow transplantation. Lancet 1 : 754-757, 1989

25. Kennedy MJ, Vogelsang GB, Beveridge RA, Farmer ER Altomonte V, Huelskamp AM, Davidson NE: Phase I trial of intravenous cyclosporine to induce graft-versus-host disease in women undergoing autologous bone marrow transplantation for breast cancer. J Clin Oncol 11: 478-484, 1993

26. Agah R, Malloy B, Kerner M, Girgis E, Bean P, Twomey P, Mazumder A: Potent graft antitumor effect in natural killer-resistant disseminated tumors by transplantation of interleukin 2-activated syngeneic bone marrow in mice. Cancer Res 49: 5959-5963, 1989

27. Giralt S, Weber D, Colome M, Dimopoulos M, Mehra R, Van Besien K, Gajewski J, Andersson B, Khouri I, Przepiorka D, von 
Wolff B, Delasalle K, Korbling M, Seong D, Alexanian R, Champlin R: Phase I trial of cyclosporine-induced autologous graft-versus-host disease in patients with multiple myeloma undergoing high-dose chemotherapy with autologous stem-cell rescue. J Clin Oncol 15: 667-673, 1997

28. Massumoto C, Benyunes MC, Sale G, Beauchamp M, York A, Thompson JA, Buckner CD, Fefer A: Close simulation of acute graft-versus-host disease by interleukin-2 administered after autologous bone marrow transplantation for hematologic malignancy. Bone Marrow Transplant 17: 351-356, 1996

29. Weiden PL, Sullivan KM, Flournoy N, Storb R, Thomas ED: Antileukemic effect of chronic graft-versus-host disease: contribution to improved survival after allogeneic marrow transplantation. N Engl J Med 304: 1529-1533, 1981

30. Jones RJ, Ambinder RF, Piantadosi S, Santos GW: Evidence of a graft-versus-lymphoma effect associated with allogeneic bone marrow transplantation. Blood 77: 649-653, 1991

31. Keever CA, Pekle K, Gazzola MV, Collins NH, Gillio A: NK and LAK activities from human marrow progenitors. I The effects of interleukin-1 and interleukin-2. Cell Immunol 126: 211-226, 1990

32. Charak BS, Agah R, Gray D, Mazumder A: Interaction of various cytokines with interleukin 2 in the generation of killer cells from human bone marrow: application in purging of leukemia. Leuk Res 15: 801-810, 1991

33. Charak BS, Choudhary GD, Tefft M, Mazumder A: Interleukin-2 in bone marrow transplantation: preclinical studies. Bone Marrow Transplant 10: 103-111, 1992

34. Charak BS, Agah R, Brynes RK, Chogyoji M, Groshen S, Chen SC, Mazumder A: Interleukin-2 (IL-2) and IL-2-activated bone marrow in transplantation: evaluation from a clinical perspective. Bone Marrow Transplant 9: 479-486, 1992

35. Charak BS, Brynes RK, Groshen S, Chen SC, Mazumder A: Bone marrow transplantation with interleukin-2-activated bone marrow followed by interleukin-2 therapy for acute myeloid leukemia in mice. Blood 76: 2187-2190, 1990

36. Verma UN, Bagg A, Brown E, Mazumder A: Interleukin-2 activation of human bone marrow in long-term cultures: an effective strategy for purging and generation of anti-tumor cytotoxic effectors. Bone Marrow Transplant 13: 115-123, 1994

37. Meehan KR, Ballen K, Boccia R: A multicenter phase I trial using STAMP V (CTCb) with IL-2 activated PBSC and IL-2 post transplantation for women with high-risk breast cancer. Blood 92: abs, 1994

38. Herrera C, Garcia-Perez MJ, Ramirez R, Martin C, Alvarez MA, Martinez F, Gomez P, Garcia-Castellano JM, Torres A: Lymphokine-activated killer (LAK) cell generation from peripheral blood stem cells by in vitro incubation with low-dose interleukin-2 plus granulocyte-macrophage colony-stimulating factor. Bone Marrow Transplant 19: 545-551, 1997

39. Meehan KR, Slack R, Gehan E, Herscowitz HB, Areman EM, Ebadi M, Cairo MS, Lippman ME: Mobilization of peripheral blood stem cells with paclitaxel and rhG-CSF in high-risk breast cancer patients. J Hematother Stem Cell Res 11: 415-421, 2002

40. Pittet MJ, Speiser DE, Valmori D, Cerottini JC, Romero P: Cutting edge: cytolytic effector function in human circulating CD8 + $\mathrm{T}$ cells closely correlates with CD56 surface expression. J Immunol 164: 1148-52, 2000

41. Zoll B, Lefterova P, Csipai M, Finke S, Trojaneck B, Ebert O, Micka B, Roigk K, Fehlinger M, Schmidt-Wolf GD, Huhn D, Schmidt-Wolf IG: Generation of cytokine-induced killer cells using exogenous interleukin-2, -7 or -12 . Cancer Immunol Immunother 47: 221-226, 1998

42. Schmidt-Wolf GD, Negrin RS, Schmidt-Wolf IG: Activated T cells and cytokine-induced $\mathrm{CD} 3+\mathrm{CD} 56+$ killer cells. Ann Hematol 74: 51-56, 1997

43. Crown J: Smart bombs versus blunderbusses: high-dose chemotherapy for breast cancer. Lancet 364: 1299-1300, 2004

Address for offprints and correspondence: Claudine Isaacs, MD, Lombardi Comprehensive Cancer Center, Breast Cancer Program, 3800 Reservoir Rd, NW, Washington, DC 20057; Tel.: (202) 444-3677; Fax: (202) 444-9429; E-mail: isaacsc@georgetown.edu 